\title{
What determines the thickness of a biological membrane
}

\author{
Norbert Kučerka1 ${ }^{1,2}$, Mu-Ping Nieh ${ }^{1}$, Jeremy Pencer ${ }^{3}$, Jonathan N. Sachs ${ }^{4}$ and John Katsaras ${ }^{1,5,6}$ \\ ${ }^{1}$ Canadian Neutron Beam Centre, National Research Council, Chalk River, Ontario K0J 1P0, Canada \\ 2 Department of Physical Chemistry of Drugs, Comenius University, 83232 Bratislava, Slovakia \\ ${ }^{3}$ Atomic Energy of Canada Limited, Chalk River Laboratories, Chalk River, Ontario K0J 1P0, Canada \\ ${ }^{4}$ Biomedical Engineering, University of Minnesota, Minneapolis, Minnesota 55455, USA \\ ${ }^{5}$ University of Guelph, Guelph, Ontario N1G 2W1, Canada \\ ${ }^{6}$ Department of Physics, Brock University, 500 Glenridge Avenue, St. Catharines, Ontario L2S 3A1, Canada
}

\begin{abstract}
Membrane thickness is thought to play a key role in protein function. Thus understanding the cell's ability to modulate the thickness of its membranes is essential in elucidating the structure/ function relationship in biological membranes. We have investigated the influence of cholesterol on the structure of "thin" (diC14:1PC) and "thick" (diC22:1PC) phospholipid bilayers using oriented multibilayers and small angle neutron diffraction. Neutron contrast variation was used to determine the structure factors and the distribution of water across the bilayers. We found that in response to cholesterol, bilayer thickness changed in a similar fashion in both systems. The thickening of bilayers was rationalized in terms of cholesterol's ordering effect on the lipid's acyl chains, which dominates over the other option of rectifying the hydrophobic mismatch, surprisingly even in the case of diC22:1PC and cholesterol.
\end{abstract}

Key words: Phospholipid bilayer - Cholesterol - Bilayer thickness - Neutron diffraction - Contrast variation

\section{Introduction}

The thickness of biological membranes is known to vary, though one exciting speculation is that it acts as a stimulus for membrane proteins. The insertion and orientation of polypeptides, as well as the activity of integral membrane proteins critically depends on bilayer thickness (Lee 2004). On the other hand, because of the structural flexibility of lipid hydrocarbon chains, a membrane can adjust its thickness in order to minimize unfavourable thermodynamic interactions between water and hydrophobic protein surfaces, a process known as hydrophobic matching (Harroun et al. 1999). An example of this is sarcoplasmic reticulum $\mathrm{Ca}^{2+}$. transporting ATPase reconstituted into bilayers made up of monounsaturated phospholipids and a biological detergent (Karlovská et al. 2006). Enzymatic activity was maximal in bilayers composed of medium-length (18 carbons) lipids,

Correspondence to: Norbert Kučerka, National Research Council, Chalk River, Ontario K0J 1P0, Canada

E-mail: norbert.kucerka@nrc.gc.ca and decreased as much as four-fold, in both short- (14 carbons) and long-chain (22 carbons) lipid bilayers.

Based on its ubiquitous presence in animal cell membranes, it has long been assumed that the dominant player in determining membrane thickness is cholesterol (Bretscher and Munro 1993). The activity of Na,K-ATPase was shown to be sensitive not only to phospholipid chain length, but also to cholesterol content (Cornelius 2001). Maximal protein activation was seen in long-chain (22 carbon) phospholipids in the absence of cholesterol, and medium-chain (18 carbon) phospholipids in the presence of $40 \mathrm{~mol} \%$ cholesterol. This suggests that cholesterol increased the thickness of the shorter chain bilayer such that it was comparable to the thickness of the pure long-chain bilayer assuming that the hydrophobic matching between the lipid and protein dominates the free energy of activation. Having a basic understanding of how a membrane modulates its thickness is thus essential in understanding the structure/function relationship in biological membranes.

In general, it is assumed that bilayer thickness is a key factor for proper protein function. However, the acyl's chain degree of unsaturation also has a marked effect on bilayer 
properties and protein-lipid interactions. For example, in mixtures of saturated and unsaturated lipids, cholesterol partitions preferably within saturated lipids, increasing lipid acyl chain order and concomitantly, bilayer thickness. Such results were reported for egg lecithin (Levine and Wilkins 1971), a wide range of saturated lipids (McIntosh 1978; Gallová et al. 2004b), and bilayers made up of unsaturated lipids (Gallová et al. 2004a, 2008; Kučerka et al. 2007b, 2008b). On the other hand, a decrease in bilayer thickness was reported for gel phase, long-chain saturated lipids (McIntosh 1978), and recent polyunsaturated lipid (diC20:4PC)/cholesterol data show a thinning of the membrane, with cholesterol being sequestered in the bilayer centre (Harroun et al. 2008). Finally, the additional effect of bilayer asymmetry - caused most likely by bilayer curvature - was recently observed in the case of short mono-unsaturated (diC14:1PC) bilayers (Kučerka et al. 2008b).

Although these results might at first seem inconclusive, this is not necessarily the case. Reasons for the discrepancies between the various studies may be attributed to a number of factors. For example, differences in the thermodynamic state of lipid bilayers, in particular, the level of hydration may be a root cause for many of these discrepancies. In order to increase spatial resolution, many diffraction studies have been performed on less than fully hydrated bilayers, as water tends to increase bilayer fluctuations. Another factor influencing bilayer structure is its geometry. Although recent studies of single species zwitterionic lipids have reported no effect as a result of bilayer curvature (Kučerka et al. 2007c), in the case of charged lipid bilayers, curvature resulted in the formation of asymmetric membranes (Brzustowicz and Brunger 2005; Kučerka et al. 2007c). This was also found to be the case in lipid mixtures with cholesterol (Kučerka et al. $2008 \mathrm{~b}$ ) and the formation of domains in ternary mixtures (Pencer et al. 2008). Finally, although the use of scattering techniques has proven to be ubiquitous in structural biology, biophysics and materials science (Kučerka et al. 2007a), the differences in sensitivity between small angle scattering vs. diffraction, and X-rays vs. neutrons, have sometimes led to different conclusions regarding membrane structure (Kučerka et al. 2008a).

The addition of cholesterol into "thin" and "thick" bilayers can elucidate some of the issues regarding the membrane's response to the hydrophobic mismatch between lipids and cholesterol. To address this question, we have recently performed systematic studies on diC14:1PC and diC22:1PC lipid bilayers using fully hydrated unilamellar vesicles (ULVs), which were interrogated using small angle neutron (Kučerka et al. 2007b), and X-ray scattering, combined with small angle X-ray diffraction from oriented multilayers, including molecular dynamics (MD) simulations (Kučerka et al. 2008b). Here we complement these previous studies with small angle neutron diffraction from oriented stacks of partially dehydrated bilayers. Contrast variation is used to determine the structure factors, and ultimately the distribution of water across bilayers. We compare relative changes in bilayer thickness upon the addition of cholesterol to previously reported results (Kučerka et al. 2007b, 2008b). Consistently, we find that cholesterol increases the thickness of both systems, and for all of the different experimental conditions.

\section{Materials and Methods}

\section{Sample preparation}

Synthetic 1,2-dimyristoleoyl-sn-glycero-phosphatidylcholine (diC14:1PC) and 1,2-dierucoyl-sn-glycero-phosphatidylcholine (diC22:1PC) were purchased from Avanti Polar Lipids Inc. (Alabaster, AL) and used without further purification. Cholesterol was obtained from Sigma-Aldrich (St. Louis, MO). All other chemicals were of reagent grade. Approximately $15 \mathrm{mg}$ of pure lipid or a mixture with 40 mol\% cholesterol (thin film thickness of $\sim 0.001 \mathrm{~cm}$ when spread onto a $25 \times 60 \mathrm{~mm}^{2}$ silicon wafer) was solubilized in trifluoroethanol : methanol ( $1: 1$ by volume) in a glass vial. Each sample was deposited onto a silicon wafer and rocked during evaporation of the organic solvent in a glove box (Tristram-Nagle 2007). The samples were dried in air on a lab bench and subjected to a few annealing cycles (temperature variation between 4 and $60^{\circ} \mathrm{C}$, while in a humid environment) prior to the measurements.

\section{Neutron diffraction}

Neutron diffraction experiments were performed using the N5 triple-axis spectrometer located at the National Research Universal reactor (Chalk River Laboratories, Canada). A pyrolytic-graphite (PG) monochromator was used to select $2.37 \AA$ neutrons, while a PG filter was used to eliminate higher order reflections (i.e., $\lambda / 2, \lambda / 3$, etc.). Samples were placed in an airtight sample cell (Katsaras and Watson 2000) and hydrated to the requisite relative humidity $(\mathrm{RH})$ using a series of $\mathrm{D}_{2} \mathrm{O} / \mathrm{H}_{2} \mathrm{O}$ mixtures (i.e., $100,50,10$, and $\left.0 \% \mathrm{D}_{2} \mathrm{O}\right)$. $\mathrm{RH}$ was controlled by saturating the various solutions with $\mathrm{K}_{2} \mathrm{SO}_{4}(97 \% \mathrm{RH})$ at $25^{\circ} \mathrm{C}$. At this $\mathrm{RH}$ and temperature, all samples form liquid-crystalline bilayers.

The stability of the experimental conditions over the data collection period was confirmed by the reproducibility of the diffraction data, whereby lamellar repeat spacings and peak intensities remained constant, indicating that the bilayer structure was unaltered. Alignment quality for each sample was assessed using the Gaussian width of rocking curves (i.e., sample rotated at a fixed detector angle). With 
the exception of diC14:1PC bilayers (Figure 1), all of the samples show a sharp peak (mosaic spread of Gaussian width $0.03^{\circ}$ ) corresponding to large lateral domains of highly oriented multibilayers, most likely bilayers close to the substrate (Als-Nielsen and McMorrow 2001). This narrow peak sits atop of a broad peak (Gaussian width $0.3^{\circ}$ ) consisting of scattering from much smaller domains oriented in the same direction, but with a broader distribution of orientations (Als-Nielsen and McMorrow 2001). The lack of a central sharp peak in the case of diC14:1PC hydrated multilayers suggests a sample with numerous small domains.

\section{Experimental data treatment}

The diffraction peaks obtained from $\theta-2 \theta$ scans, where $\theta$ and $2 \theta$ are the angles of the sample and the detector, respectively, were fitted to Gaussians using an additional second-order polynomial function describing the background. Integrated intensities for the different quasi-Bragg peaks were corrected for incident flux $\left(C_{f l u x}\right)$, sample absorption $\left(C_{a b s}\right)$, and the Lorentz correction $\left(C_{\text {Lor }}\right)$, according to well-established procedures (Harroun et al. 2008). Briefly, $C_{f l u x}$ is the correction for the variation in neutron flux impinging on the sample at different $\theta$ s and it is calculated as the convolution of the beam shape (Gaussian) with the sample (step function), yielding the error function (erf) correction

$$
C_{f l u x}=1 / \operatorname{erf}\left(\frac{L \sin (\theta)}{\sqrt{8} \sigma}\right)
$$

where $L$ is the sample length and $2 \sigma$ is the beam width. $C_{a b s}$ was used to correct for the absorption of neutrons by the sample as

$$
C_{a b s}=\frac{2 \mu t / \sin (\theta)}{1-\exp (-2 \mu t / \sin (\theta))}
$$

where the sample thickness $t$ is estimated from the amount of lipid used to make the sample $(\sim 0.001 \mathrm{~cm})$, and the absorption coefficient $\mu\left(\sim 4-6 \mathrm{~cm}^{-1}\right)$ is calculated considering all of the molecules in the unit cell occupying a volume of $V_{U C}$. This is expressed as

$$
\mu=\frac{\sum N_{i} \sigma_{i}}{V_{U C}}=N_{A} \frac{\sum N_{i} \sigma_{i}}{\sum N_{i} M_{i} / \rho_{i}}
$$

where $\sigma$ is the total neutron cross section - sum of the coherent and incoherent scattering cross sections and the absorption cross section (Sears 1992), $M$ is the molecular weight and $\rho$ is the mass density of molecules in unit cell. We define the unit cell on a per lipid basis i.e., $N_{\text {lipid }}=1$ and $N_{\text {cholesterol }}=0.67$ for samples with $40 \mathrm{~mol} \%$ cholesterol.

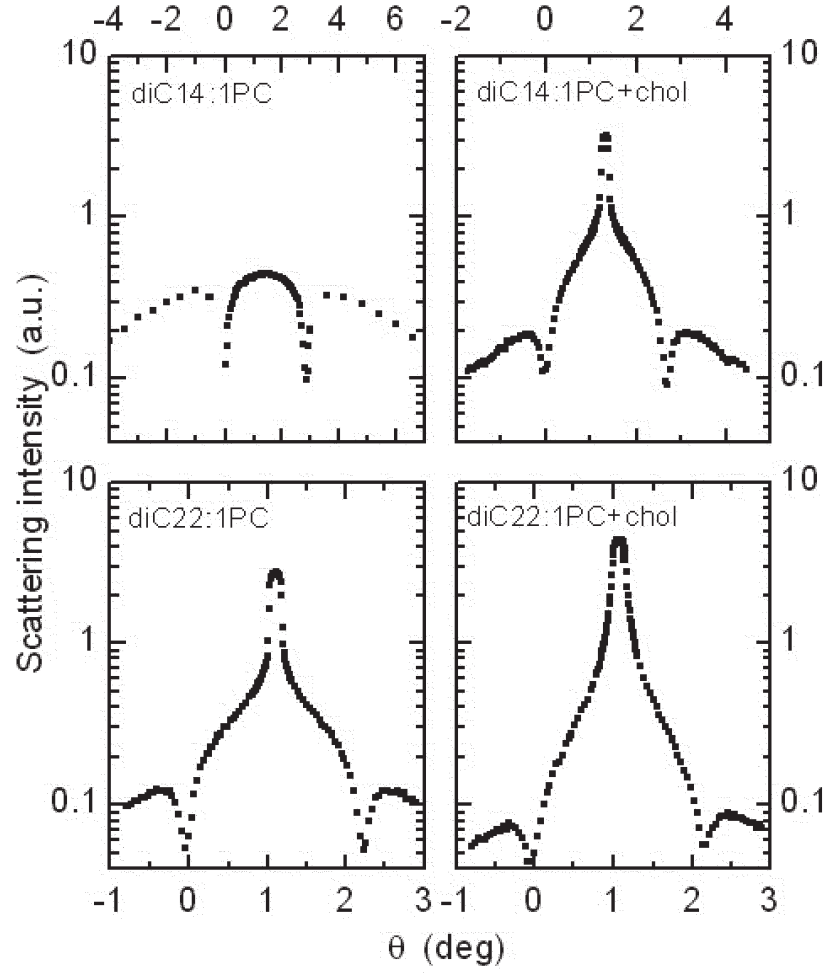

Figure 1. Semi-log plots of first-order diffraction peak rocking curves from aligned multibilayers, without and with cholesterol (chol). The width of these peaks is a good indicator of the sample's orientation with respect to the substrate. The minima along the scattering curves are the result of increased neutron absorption, which occurs when the specimen is rotated $(\theta)$ so that either the incident beam or the diffracted beam is parallel to the substrate.

Finally, the Lorentz factor for the angular velocity correction is applied as

$$
C_{\text {Lor }}=\sin (2 \theta)
$$

The amplitude for the various order $h$ scattering form factors are then calculated from the measured scattering intensities as

$$
\left|F_{h}\right|=\sqrt{C_{f l u x} C_{a b s} C_{L o r} I_{h}}
$$

\section{Determination of scattering phases}

The standard method for determining form factor phases from neutron scattering experiments is through the isotopic replacement of $\mathrm{H}_{2} \mathrm{O}$ for $\mathrm{D}_{2} \mathrm{O}$, whereby the scattering form factors measured at different contrast conditions change linearly as a function of $\mathrm{D}_{2} \mathrm{O}$ content (Worcester and Franks 1976). However, this simple approach does not determine the sign of the line's slope. For unambiguous phase deter- 
mination, we employ the procedure suggested by Leonard et al. (2001). Accordingly, we define the slope of a line for a given order $h$ obtained from a series of $\mathrm{H}_{2} \mathrm{O} / \mathrm{D}_{2} \mathrm{O}$ experiments as

$$
p(h) \equiv F\left(h, D_{2} O\right)-F\left(h, H_{2} O\right)
$$

which in terms of scattering length densities is equal to

$$
p(h)=\int_{-D / 2}^{D / 2} \rho_{W}(z) \cos \left(\frac{2 \pi h z}{D}\right) d z
$$

where $D$ is the repeat spacing corresponding to the size of the unit cell, and $\rho_{w}(z)$ is a difference between the scattering length density (SLD) distribution for bilayers measured in $\mathrm{D}_{2} \mathrm{O}$ minus the ones in $\mathrm{H}_{2} \mathrm{O}$. Since, for the most part, the SLD distribution of lipid molecules does not change with the $\mathrm{D}_{2} \mathrm{O} / \mathrm{H}_{2} \mathrm{O}$ replacement, $\rho_{w}(z)$ corresponds to the SLD distribution of water molecules, only. This distribution can be approximated by the sum of two classical error functions (Klauda et al.2006) placed at the water/bilayer interface (i.e., $\left.\pm D_{B} / 2= \pm\left(D-D_{W}\right) / 2\right)$, where $D_{B}$ and $D_{W}$ are the thicknesses of the bilayer and water layer, respectively $\left(D=D_{B}+D_{W}\right)$. Note, that the bilayer centre is placed at $z=0 \AA$. The integration of Eq. (7) then yields

$$
\begin{aligned}
& p(h)=(-1)^{h} \frac{D\left(\rho_{D_{2} O}-\rho_{H_{2} O}\right)}{\pi h} \sin \left(\frac{\pi h D_{W}}{D}\right) \\
& \exp \left(-2\left(\frac{\pi h \sigma_{W}}{D}\right)^{2}\right)
\end{aligned}
$$

where $\left(\rho_{D_{2} \mathrm{O}-} \rho_{\mathrm{H}_{2} \mathrm{O}}\right)$ is the SLD difference between $\mathrm{D}_{2} \mathrm{O}$ and $\mathrm{H}_{2} \mathrm{O}$, and $\sigma_{w}$ is the width of the water probability error function. It is worth noting that from Eq. (8) it is evident that the often-used rule of alternating slope signs (first factor) is modified by the sign of the sine function, which depends on the interlamellar water layer $\left(D_{W} / D\right)$.

\section{Scattering length density profiles}

The SLD profiles are related to the scattering form factors through their Fourier inversion. The water subtracted SLD distribution $\left(\Delta \rho(z)=\rho(z)-\rho_{w}\right)$ is expressed as

$$
\Delta \rho^{a b s}(z)=\frac{1}{D} F_{0}^{a b s}+\frac{2}{D} \sum_{h=1} F_{h}^{a b s} \cos \left(\frac{2 \pi h z}{D}\right)
$$

where $F_{h}{ }^{a b s}$ are the scattering form factors on an absolute scale. However, the experimentally determined form factors usually involve an order-independent scaling factor $\left(F_{h}=k F_{h}^{a b s}\right)$, providing only the ratios between different orders. Moreover, $F_{0}$ - corresponding to the forward scat- tering - cannot be measured experimentally. As a result, the bilayer's SLD profile calculated from only the experimentally obtained form factors involves both an additive offset, as well as a multiplicative factor. In order to place the data on an absolute scale, additional information and assumptions are needed.

The SLD's offset is directly related to $F_{0}{ }^{a b s}$, which was calculated in a manner similar to what was done previously for X-ray scattering (Nagle and Wiener 1989)

$$
A F_{0}^{a b s}=b_{L}-\rho_{W} V_{L}
$$

Here, $b_{L}$ represents the neutron scattering length (Sears 1992) of lipid moieties whose volume $V_{L}$ displaces an equivalent volume of water molecules with a neutron $\operatorname{SLD} \rho_{w}$. All of the parameters on the right side of the equation are well known, while lipid area $A$ needs to be determined. Although a value of $\sim 50-60 \AA^{2}$ is generally used for $A$, it can also be estimated for different lipids using their fully hydrated area values (Kučerka et al. 2008b) and the definition for lateral compressibility (Rand and Parsegian 1989)

$$
A=A_{100}-A_{100} D_{w} P / K_{A}
$$

where $A_{100}$ is the area per lipid and $D_{w}$ is the interlamellar water space at $100 \% \mathrm{RH}$, and $K_{A}$ is area compressibility. For the latter we assume a value of $263 \mathrm{mN} / \mathrm{m}$ for both lipids studied here - this is reasonable given the fact that this value is, for the most part, independent of lipid chain length and degree of unsaturation (Rawicz et al. 2000). $D_{w}$ is calculated as the difference between the $D$ of fully hydrated multilayers and the bilayer thickness (Kučerka et al. 2005, 2008 b), and ranges from 20 to $25 \AA$. Finally, the induced osmotic pressure $P$ at $97 \% \mathrm{RH}$ - the decrease in $D$ is less than $9 \AA-$ is $\sim 10^{6} \mathrm{~Pa}$ (Rand and Parsegian 1989; Petrache et al. 1998). Table 1 summarizes the values of the area/lipid estimated for bilayers hydrated at $97 \% \mathrm{RH}$, as well as values for the other bilayer parameters.

The scaling factor $k$ is determined by requiring that the SLD profile possesses the correct value at chosen points along the bilayer. For fully hydrated bilayers the obvious choice is to constrain the SLD profile edges to the SLD value of the solvent. However, in the case of partially dehydrated bilayers, where the water layer may not be as distinct, a more appropriate constraint is the central trough $\rho_{T}$. The last column in Table 1 shows these values as determined from recent MD simulations (Kučerka et al. 2008b). The scaling factor $k\left(F_{h}=\right.$ $\left.k F_{h}{ }^{a b s}\right)$ is then expressed as

$$
k=\frac{2 / D \sum_{h=1} F_{h}}{\Delta \rho_{T}-F_{0}^{a b s} / D}
$$


Table 1. Bilayer structural parameters. Repeat spacing $D$ and area per lipid $A$ at 100 and $97 \% \mathrm{RH}$, interlamellar water space $D_{w}$ at $100 \%$ $\mathrm{RH}$, and SLD of central $\rho_{T}$ for the various systems

\begin{tabular}{|l|c|c|c|c|c|c|}
\hline Lipid & $D_{100}(\AA)$ & $A_{100}\left(\AA^{2}\right)$ & $D_{w}(\AA)$ & $D_{97}(\AA)$ & $A_{97}\left(\AA^{2}\right)$ & $\rho_{T}\left(10^{-6} \AA^{-2}\right)$ \\
\hline diC14:1PC & $51^{\mathrm{a}}$ & $70.2^{\mathrm{a}}$ & 20.3 & 46.9 & 69.7 & -0.16 \\
\hline diC14:1PC+cholesterol & $58^{\mathrm{a}}$ & $91.3^{\mathrm{a}}$ & 25.1 & 51.3 & 90.4 & -0.19 \\
\hline diC22:1PC & $70^{\mathrm{b}}$ & $68.3^{\mathrm{a}}$ & 25.4 & 61.3 & 67.6 & -0.45 \\
\hline diC22:1PC+cholesterol & $73^{\star}$ & $81.7^{\mathrm{a}}$ & 25.4 & 64.3 & 80.9 & -0.47 \\
\hline
\end{tabular}

${ }^{\mathrm{a}}$ and ${ }^{\mathrm{b}}$ values were taken from Kučerka et al. (2008b) and Kučerka et al. (2005), respectively, while ${ }^{*}$ was estimated from $D_{97} . D_{w}$ was calculated as the difference between $D_{100}$ and lipid bilayer thicknesses found in Kučerka et al. (2008b).

and the absolute scale SLD profile is calculated from the experimental $F_{h}$ as

$$
\Delta \rho^{a b s}(z)=\frac{1}{D} F_{0}^{a b s}+\frac{1}{k} \frac{2}{D} \sum_{h=1} F_{h} \cos \left(\frac{2 \pi h z}{D}\right)
$$

\section{Water probability profiles}

The distribution of water can be obtained from difference SLD profiles calculated from contrast varied experimental data. Each SLD can be parsed into contributions corresponding to the bilayer (i.e., lipid plus cholesterol) and water molecules as follows

$$
\rho(z)=\rho_{B} P_{B}(z)+\rho_{W} P_{W}(z)
$$

where $\rho_{B}$ and $\rho_{w}$ are the mean SLDs of the bilayer and water, respectively, and $P_{B}(z)$ and $P_{W}(z)$ are their probabilities. The latter are contrast independent, universal probabilities that satisfy the spatial conservation principle (i.e., $P_{B}(z)+$ $P_{W}(z)=1$ ) at each point $z$ along the bilayer (Kučerka et al. 2008a). Since $\rho_{B}$ does not depend on external contrast conditions, the subtraction of SLD distributions measured at the two different $\mathrm{D}_{2} \mathrm{O}$ contents includes only the probability of water, which can be expressed as

$$
P_{W}(z)=\frac{\rho_{1}(z)-\rho_{2}(z)}{\rho_{W 1}-\rho_{W 2}}
$$

The water probability $P_{W}(z)$ is between 0 and 1 if $\rho_{1}(z)$ and $\rho_{2}(z)$ are obtained on an absolute scale. Finally, $P_{B}(z)$ can then be calculated using the complementarity principle (i.e., $1-P_{W}(z)$ ).

\section{Results and Discussion}

Up to seven orders of Bragg diffraction were obtained for the different samples and contrast conditions. Changes in contrast resulted in changes to the scattered intensities (Figure 2). Also apparent from Figure 2 is that the position of the various orders shifted for the different samples, as a result of differences

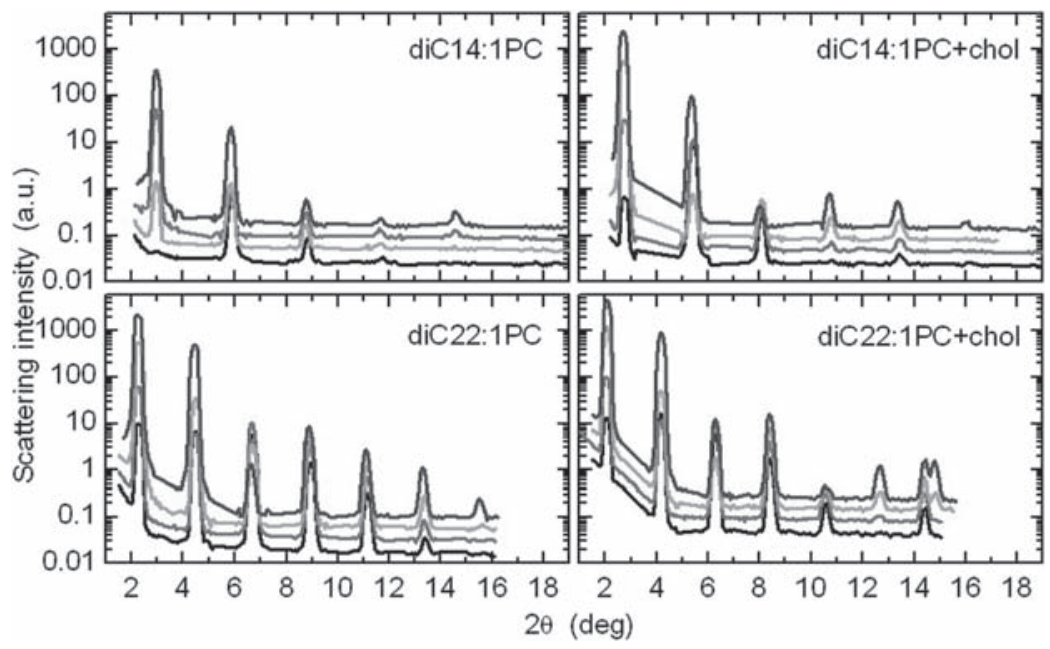

Figure 2. Diffraction curves collected for various samples measured at four different contrast conditions. The curves were shifted vertically for clarity. From bottom to top they correspond to $0,10,50$ and $100 \% \mathrm{D}_{2} \mathrm{O}$ solvent. 
in their repeat spacings. The thinner lipid bilayers (diC14:1PC) were measured over an extended $2 \theta$ range making sure that no Bragg peaks were overlooked, as the peaks are spread over a greater $2 \theta$. For example, the third order Bragg peak for diC14:1PC corresponds to the fourth peak of the diC22:1PC lipid. As a result, for thinner bilayers a rapid decrease in the signal-to-noise ratio at higher scattering angles allowed for the observation of fewer Bragg peaks. Moreover, the poorer quality of the diC14:1PC sample (see Figure 1) also contributed to fewer Bragg peaks being observed.

The corrected scattering amplitudes were used in determining the phases, as described in Materials and Methods section. The strong scattered intensities from the thicker bilayers resulted in the straightforward assignment of phases, while pure diC14:1PC proved to be more problematic. Nevertheless, in the case of diC14:1PC bilyers their structure was determined, for the most part, by the dominant scattering arising from the first three Bragg peaks, while only subtle effects were observed when varying the signs of the higher order peaks. Figure 3 shows the graphs used to determine the slopes defined according to Eq. (8). From these plots it can be seen that the sign of the slope alternates for the first 3-4 orders. This is in agreement with the theoretical evaluation by Leonard et al. (2001), showing that the signs alternate for ap-

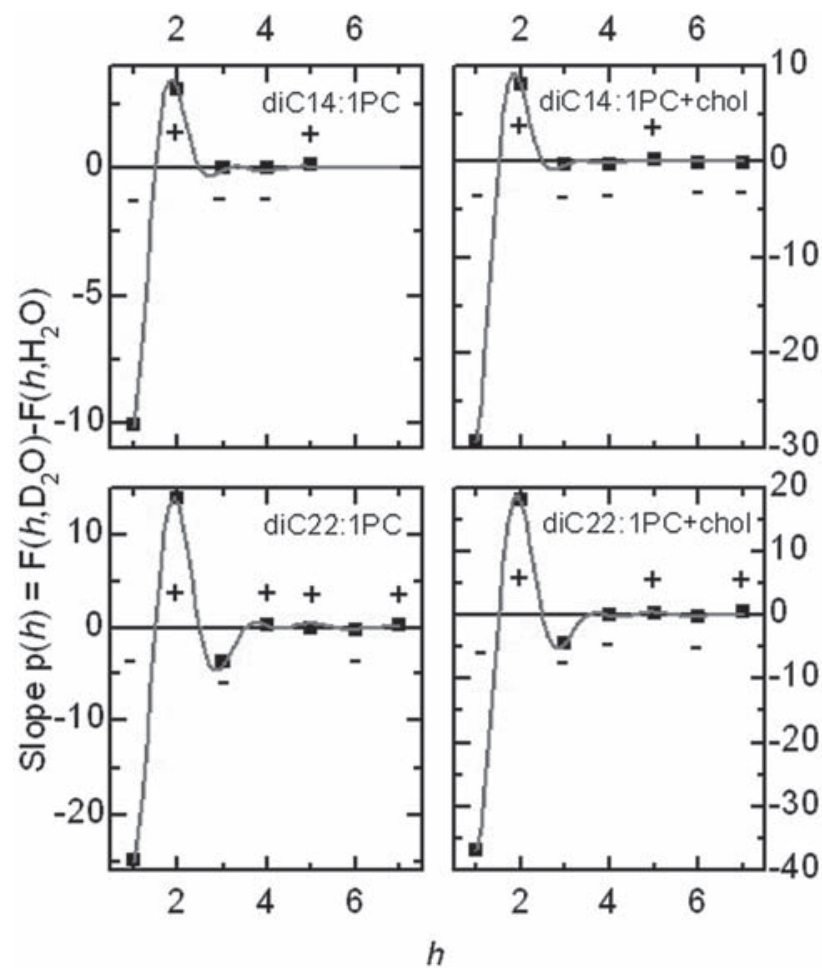

Figure 3. Plot of the slopes, $p(h)$, as a function of diffraction order, $h$, according to Eq. (8). The sign symbols emphasize the sign of the slopes obtained from the fits.
Table 2. Parameters obtained from the analysis based on Eq. (8)

\begin{tabular}{|l|c|c|c|}
\hline Lipid & $D_{w}(\AA)$ & $\sigma_{w}(\AA)$ & $D_{B}(\AA)$ \\
\hline diC14:1PC & $15.1 \pm 0.7$ & $4.6 \pm 0.3$ & $31.8 \pm 0.7$ \\
\hline diC14:1PC+cholesterol & $16.1 \pm 0.4$ & $5.6 \pm 0.2$ & $35.2 \pm 0.4$ \\
\hline diC22:1PC & $14.9 \pm 0.9$ & $4.3 \pm 0.4$ & $46.4 \pm 0.9$ \\
\hline diC22:1PC+cholesterol & $16.3 \pm 0.5$ & $5.0 \pm 0.2$ & $48.0 \pm 0.5$ \\
\hline
\end{tabular}

$D_{W}$ is the water layer thickness and $\sigma_{w}$ is the characteristic width of the bilayer-water interface. $D_{B}$ is the bilayer thickness and is calculated from $D-D_{W}$.

proximately the first 10 orders in rather dehydrated samples (i.e., reduced $D_{W}$ ), while this rule breaks down after only 3 orders in the case of much more hydrated samples.

Table 2 presents the different structural parameters obtained using Eq. (8). Comparison of the water layer thickness $D_{W}$ of multilayers at $97 \% \mathrm{RH}$ with those at $100 \% \mathrm{RH}$ (see Table 1), suggests that the change in the repeat spacing $D$ is almost entirely compensated by changes in the water layer. This result is consistent with our calculations (see Materials and Methods) that showed that partial dehydration (i.e., 97\% RH) of bilayers has no effect, for the most part, on lipid area.

The signs of the slopes obtained using Eq. (8) are used to assign the signs of the form factors. This is illustrated for the diC22:1PC sample in Figure 4, while Table 3 summarizes all of the corrected scattering form factors along with their phases.

The corrected scattering form factors were subsequently Fourier transformed into neutron SLD profiles using Eq. (13).

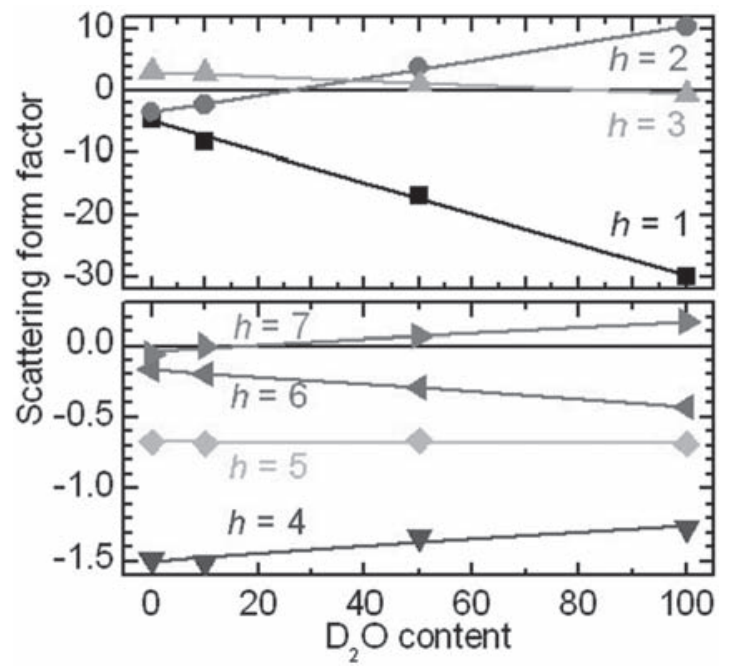

Figure 4. Linear dependence of the scattering form factors as a function of $\mathrm{D}_{2} \mathrm{O}$ content for seven diffraction orders, $h$, for diC22:1PC bilayers. The sign of the slopes were determined from the plot in Figure 3 and used to determine the phases of the different form factors. 
Table 3. Corrected experimental form factors

\begin{tabular}{|c|c|c|c|c|c|c|c|c|}
\hline \multirow[t]{2}{*}{ Sample } & \multirow{2}{*}{$\begin{array}{c}\mathrm{D}_{2} \mathrm{O} \\
\text { content }\end{array}$} & \multicolumn{7}{|c|}{ Diffraction peak order } \\
\hline & & 1 & 2 & 3 & 4 & 5 & 6 & 7 \\
\hline \multirow[t]{4}{*}{ diC14:1PC } & 100 & -10.2 & 1.88 & 0.263 & -0.112 & 0.173 & 0 & 0 \\
\hline & 50 & -4.13 & 0.503 & 0.269 & -0.121 & 0.114 & 0 & 0 \\
\hline & 10 & -0.96 & -0.931 & 0.281 & -0.109 & 0.0518 & 0 & 0 \\
\hline & 0 & 0 & -1.12 & 0.297 & -0.0985 & 0.0421 & 0 & 0 \\
\hline \multirow{4}{*}{$\begin{array}{l}\text { diC14:1PC } \\
+ \text { cholesterol }\end{array}$} & 100 & -31.0 & 4.33 & 0.235 & -0.335 & 0.263 & -0.0964 & -0.0559 \\
\hline & 50 & -16.3 & 0.487 & 0.413 & -0.242 & 0.213 & -0.0196 & 0 \\
\hline & 10 & -5.36 & -2.91 & 0.599 & -0.121 & 0.163 & 0 & 0 \\
\hline & 0 & -1.08 & -3.74 & 0.607 & -0.0666 & 0.148 & 0.0638 & 0.0439 \\
\hline \multirow[t]{4}{*}{$\operatorname{diC} 22: 1 \mathrm{PC}$} & 100 & -30.0 & 10.4 & -0.484 & -1.27 & -0.686 & -0.425 & 0.167 \\
\hline & 50 & -16.8 & 3.86 & 1.20 & -1.33 & -0.66 & -0.289 & 0.0669 \\
\hline & 10 & -8.14 & -2.26 & 2.83 & -1.51 & -0.683 & -0.194 & 0 \\
\hline & 0 & -4.53 & -3.46 & 3.06 & -1.49 & -0.672 & -0.163 & -0.0541 \\
\hline \multirow{4}{*}{$\begin{array}{l}\text { diC22:1PC } \\
+ \text { cholesterol }\end{array}$} & 100 & -42.4 & 13.2 & -1.47 & -1.65 & -0.224 & -0.432 & 0.498 \\
\hline & 50 & -23.9 & 4.44 & 0.758 & -1.6 & -0.343 & -0.283 & 0.266 \\
\hline & 10 & -9.90 & -3.01 & 2.52 & -1.64 & -0.421 & -0.146 & 0.0790 \\
\hline & 0 & -4.98 & -4.98 & 2.98 & -1.53 & -0.468 & -0.081 & 0.0716 \\
\hline
\end{tabular}

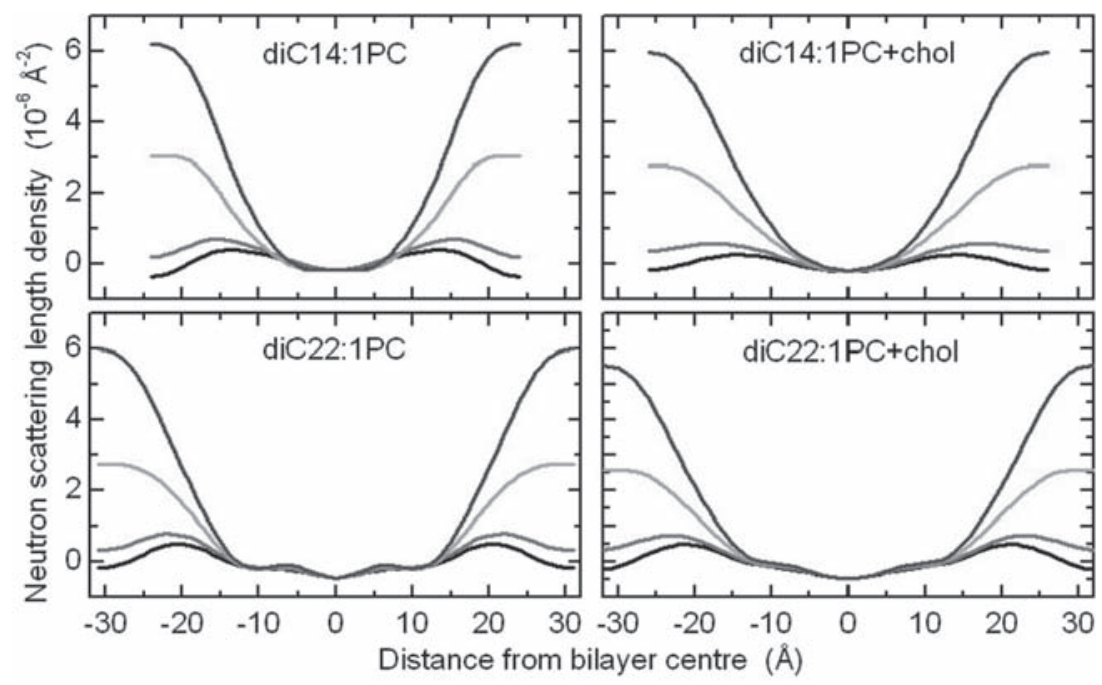

Figure 5. 1D scattering length density (SLD) profiles of diC14:1PC and diC22:1PC, without and with cholesterol (chol). From bottom to top, the SLD profiles correspond to $0,10,50$ and $100 \% \mathrm{D}_{2} \mathrm{O}$ solvents.

Figure 5 shows that the fewer diffraction peaks observed from diC14:1PC bilayers, with and without cholesterol, yielded lower resolution SLD profiles. Consistently, compared to the other bilayers studied, MD simulations of diC14:1PC bilayers show that they are more disordered (Kučerka et al. 2008b), smearing the finer structural details that are clearly visible in diC22:1PC bilayers, with and without cholesterol.

The more detailed structure obtained for the thicker bilayers shows a small trough in the bilayer centre, which corre- sponds to the disordered, low SLD methyl groups. In contrast, a small hump is observed at $\sim 15 \AA$ and $\sim 17.5 \AA$ for diC14:1PC bilayers, without and with cholesterol, respectively. In the case of diC22:1PC bilayers, the equivalent feature without and with cholesterol, appears at 22 and $23 \AA$, respectively. This hump is the result of higher SLD carbonyl and phosphate groups (Kučerka et al. 2008a), and its position in all systems is in excellent agreement with recent MD simulations (Kučerka et al. 2008b). The disappearance of this feature from SLD profiles 


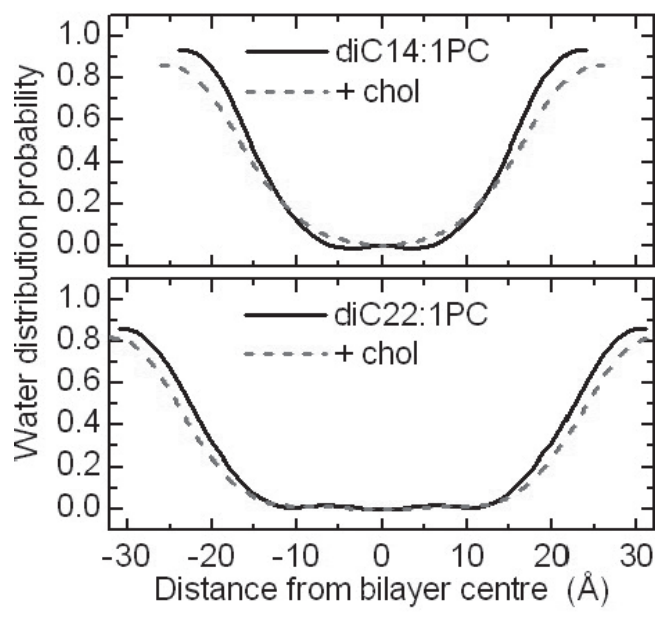

Figure 6. Water probabilities obtained from difference SLD profiles. The top panel compares diC14:1PC bilayers with and without cholesterol (chol), while the bottom panel shows the effect of chol on the long-chain diC22:1PC bilayers.

at higher $\mathrm{D}_{2} \mathrm{O}$ contents (i.e. 50 and $100 \%$ ) is due to high SLD $\mathrm{D}_{2} \mathrm{O}$ molecules penetrating the lipid bilayer.

The SLD profiles shown in Figure 5 reflect the high contrast between the fully protonated lipid and water, particularly in the case of $100 \% \mathrm{D}_{2} \mathrm{O}$. This characteristic feature of neutron scattering can be used to directly determine, to a first approximation, the distribution of water across the bilayer, and thus the bilayer thickness. As expected, diC14:1PC bilayers are clearly much thinner than diC22:1PC bilayers. More importantly, comparison of bilayers with and without cholesterol shows that the addition of cholesterol results in bilayer thickening.

This thickening is much more evident when comparing difference SLD profiles. Even though for a given system the SLD profiles obtained at the different contrast conditions look different, they all correspond to the same distribution of lipid and water molecules across the bilayer. Difference SLD profiles are then obtained from different contrast conditions according to Eq. (15). Figure 6 shows the averaged water probabilities for the different systems studied.

From the water probability one can infer the bilayer probability (i.e. lipid and cholesterol), as the two profiles (i.e. water

Table 4. Structural parameters obtained from difference SLD profiles

\begin{tabular}{|l|c|c|c|}
\hline Lipid & $D_{B}(\AA)$ & $\sigma_{w}(\AA)$ & $D_{W}(\AA)$ \\
\hline diC14:1PC & $30.4 \pm 0.8$ & $4.3 \pm 0.7$ & $16.5 \pm 0.8$ \\
\hline diC14:1PC+cholesterol & $32.0 \pm 1.0$ & $5.7 \pm 0.8$ & $19.3 \pm 1.0$ \\
\hline diC22:1PC & $43.8 \pm 1.2$ & $4.9 \pm 0.9$ & $17.5 \pm 1.2$ \\
\hline diC22:1PC+cholesterol & $46.2 \pm 0.4$ & $5.2 \pm 0.3$ & $18.1 \pm 0.4$ \\
\hline
\end{tabular}

and bilayer) are complementary (i.e. $P_{W}(z)+P_{B}(z)=1$ ). The central region consists of lipid molecules, while the probability of water achieves maximal values outside this region (Figure 6). The gradual increase from zero to one corresponds to the penetration of water into the bilayer, and its central value defines the total thickness of the bilayer. As mentioned in the Materials and Methods section, the water probability can be approximated by the sum of two classical error functions. Fitting such a function to the profiles shown in Figure 6 then provides quantitative results for the discussed structural parameters (Table 4).

Consistent with expectations and previous results (Gallová et al. 2004a; Kučerka et al. 2007b, 2008b), lipid bilayers made up of diC14:1PC are thinner than diC22:1PC bilayers (see also Table 2). However, comparison of the data in Tables 2 and 4 reveals a systematic difference between the two methods of $\sim 2 \AA$, thus providing an estimate of the systematic error that exists between reciprocal space and real space analyses. While the first method employs slopes averaged over the four different contrast conditions in reciprocal space, the second method treats the diffraction data obtained at different contrast conditions individually and averages the results in real space.

More importantly, however, the two tables show excellent agreement with regards to the effect of cholesterol. The addition of cholesterol resulted in increasing the bilayer thickness by about $2 \AA$ and widening the lipid/water interface by about $1 \AA$, regardless of bilayer thickness. Although this might at first seem surprising in the case of diC22:1PC bilayers whose hydrocarbon region is greater than the length of a cholesterol molecule, it is in agreement with previously published studies (Gallová et al. 2004a, 2008; Kučerka et al. 2008b). The widely accepted model of lipid/cholesterol interactions assumes that cholesterol affects membrane structure in two ways: due to its rigid structure, cholesterol increases lipid acyl chain order, which for highly flexible fluid phase lipid bilayers results in increased bilayer thickness. On the other hand, it is believed that a rigid molecule such as cholesterol can dictate the thickness of a bilayer's hydrocarbon chain region via hydrophobic mismatch. In the end, our results indicate that the overriding influence of cholesterol is to order the lipid's hydrocarbon chains, not to rectify the hydrophobic mismatch.

Acknowledgements. The authors thank Prof. Pavol Balgavý for many constructive discussions over the years. In particular, N. K. would like to thank Prof. Pavol Balgavý for the years of personal and scientific support, especially during the early years of N. K.'s scientific career.

\section{References}

Als-Nielsen J., McMorrow D. (2001): Elements of Modern X-Ray Physics. John Wiley\&Sons, Ltd.

Bretscher M. S., Munro S. (1993): Cholesterol and the Golgi apparatus. Science 261, 1280-1281; doi:10.1126/science.8362242 PMid:8362242 
Brzustowicz M. R., Brunger A. T. (2005): X-ray scattering from unilamellar lipid vesicles. J. Appl. Crystalogr. 38, 126-131; doi:10.1107/S0021889804029206

Cornelius F. (2001): Modulation of Na,K-ATPase and Na-ATPase activity by phospholipids and cholesterol. I. Steady-state kinetics. Biochemistry 40, 8842-8851; doi:10.1021/ bi010541g PMid:11467945

Gallová J., Uhríková D., Hanulová M., Teixeira J., Balgavý P. (2004a): Bilayer thickness in unilamellar extruded 1,2-dimyristoleoyl and 1,2-dierucoyl phosphatidylcholine vesicles: SANS contrast variation study of cholesterol effect. Colloids Surf., B 38, 11-14

Gallová J., Uhríková D., Islamov A., Kuklin A., Balgavý P. (2004b) Effect of cholesterol on the bilayer thickness in unilamellar extruded DLPC and DOPC liposomes: SANS contrast variation study. Gen. Physiol. Biophys. 23, 113-128

Gallová J., Uhríková D., Kučerka N., Teixeira J., Balgavý P. (2008) Hydrophobic thickness, lipid surface area and polar region hydration in monounsaturated diacylphosphatidylcholine bilayers: SANS study of effects of cholesterol and $\beta$-sitosterol in unilamellar vesicles. Biochim. Biophys. Acta 1778, 2627-2632; doi:10.1016/j.bbamem.2008.08.009

Harroun T. A., Heller W. T., Weiss T. M., Yang L., Huang H. W. (1999): Experimental evidence for hydrophobic matching and membrane-mediated interactions in lipid bilayers containing gramicidin. Biophys. J. 76, 937-945; doi:10.1016/ S0006-3495(99)77257-7 PMid:9929495 PMCid:1300095

Harroun T. A., Katsaras J., Wassall S. R. (2008): Cholesterol is found to reside in the center of a polyunsaturated lipid membrane. Biochemistry 47, 7090-7096; doi:10.1021/ bi800123b PMid:18543943

Karlovská J., Uhríková D., Kučerka N., Teixeira J., Devínsky F., Lacko I., Balgavý P. (2006): Influence of N-dodecyl-N,Ndimethylamine $\mathrm{N}$-oxide on the activity of sarcoplasmic reticulum $\mathrm{Ca} 2+$-transporting ATPase reconstituted into diacylphosphatidylcholine vesicles: effects of bilayer physical parameters. Biophys. Chem. 119, 69-77; doi:10.1016/j.bpc.2005.09.00 PMid:16223561

Katsaras J., Watson M. J. (2000): Sample cell capable of 100\% relative humidity suitable for $\mathrm{x}$-ray diffraction of aligned lipid multibilayers. Rev. Sci. Instrum. 71, 1737-1739; doi:10.1063/1.1150529

Klauda J. B., Kučerka N., Brooks B. R., Pastor R. W., Nagle J. F. (2006): Simulation-based methods for interpreting $\mathrm{X}$ ray data from lipid bilayers. Biophys. J. 90, 2796-2807; doi:10.1529/biophysj.105.075697 PMid:16443652 PMCid:1414576

Kučerka N., Tristram-Nagle S., Nagle J. F. (2005): Structure of fully hydrated fluid phase lipid bilayers with monounsaturated chains. J. Membr. Biol. 208, 193-202

Kučerka N., Nieh M. P., Pencer J., Harroun T., Katsaras J. (2007a): The study of liposomes, lamellae and membranes using neutrons and X-rays. Curr. Opin. in Colloid Interface Sci. 12, 17-22; doi:10.1016/j.cocis.2006.11.006

Kučerka N., Pencer J., Nieh M.-P., Katsaras J. (2007b): Influence of cholesterol on the bilayer properties of monounsaturated phosphatidylcholine unilamellar vesicles. Eur. Phys. J., E: Soft Matter 23, 247-254; doi:10.1140/epje/i2007-10202-8
Kučerka N., Pencer J., Sachs J. N., Nagle J. F., Katsaras J. (2007c): Curvature effect on the structure of phospholipid bilayers. Langmuir 23, 1292-1299; doi:10.1021/la062455t PMid: 17241048

Kučerka N., Nagle J. F., Sachs J. N., Feller S. E., Pencer J., Jackson A., Katsaras J. (2008a): Lipid bilayer structure determined by the simultaneous analysis of neutron and X-ray scattering data. Biophys. J. 95, 2356-2367; doi:10.1529/ biophysj.108.132662 PMid:18502796

Kučerka N., Perlmutter J. D., Pan J., Tristram-Nagle S., Katsaras J., Sachs J. N. (2008b): The effect of cholesterol on short- and long-chain monounsaturated lipid bilayers as determined by molecular dynamics simulations and X-ray scattering. Biophys. J. 95, 2792-2805; doi:10.1529/ biophysj.107.122465 PMid:18515383

Lee A. G. (2004): How lipids affect the activities of integral membrane proteins. Biochim. Biophys. Acta 1666, 62-87; doi:10.1016/j.bbamem.2004.05.012

Leonard A., Escrive C., Laguerre M., Pebay-Peyroula E., Neri W., Pott T., Katsaras J., Dufourc E. J. (2001): Location of cholesterol in DMPC membranes. A comparative study by neutron diffraction and molecular mechanics simulation. Langmuir 17, 2019-2030; doi:10.1021/la001382p

Levine Y. K., Wilkins M. H. (1971): Structure of oriented lipid bilayers. Nature New Biol. 230, 69-72

McIntosh T. J. (1978): The effect of cholesterol on the structure of phosphatidylcholine bilayers. Biochim. Biophys. Acta 513, 43-58; doi:10.1016/0005-2736(78)90110-4

Nagle J. F., Wiener M. C. (1989): Relations for lipid bilayers. Connection of electron density profiles to other structural quantities. Biophys. J. 55, 309-313; doi:10.1016/S00063495(89)82806-1 PMid:2713444 PMCid:1330472

Pencer J., Jackson A., Kučerka N., Nieh M. P., Katsaras J. (2008): The influence of curvature on membrane domains. Eur. Biophys. J. 37, 665-671; doi:10.1007/s00249-008-0304-1 PMid:18369611

Petrache H. I., Gouliaev N., Tristram-Nagle S., Zhang R., Suter R. M., Nagle J. F. (1998): Interbilayer interactions from high-resolution x-ray scattering. Phys. Rev. E 57, 7014; doi:10.1103/PhysRevE.57.7014

Rand R. P., Parsegian V. A. (1989): Hydration forces between phospholipid bilayers. Biochim. Biophys. Acta 988, 351-376

Rawicz W., Olbrich K. C., McIntosh T. J., Needham D., Evans E. (2000): Effect of chain length and unsaturation on elasticity of lipid bilayers. Biophys. J. 79, 328-339; doi:10.1016/S00063495(00)76295-3 PMid:10866959 PMCid:1300937

Sears V. F. (1992): Neutron scattering lengths and cross sections. Neutron News 3, 26-37; doi:10.1080/10448639208218770

Tristram-Nagle S. (2007): Preparation of oriented, fully hydrated lipid samples for structure determination using X-ray scattering. Methods Mol. Biol. 400, 63-75

Worcester D. L., Franks N. P. (1976): Structural analysis of hydrated egg lecithin and cholesterol bilayers. II. Neutron diffraction. J. Mol. Biol. 100, 359-378; doi:10.1016/S00222836(76)80068-X PMid:943549

Received: September 23, 2008

Final version accepted: November 3, 2008 